\title{
Fabrication of Colloidal Laves Phases via Hard Tetramers and Hard Spheres: Bulk Phase Diagram and Sedimentation Behavior
}

\author{
Guido Avvisati, ${ }^{\circledR}$ Tonnishtha Dasgupta, and Marjolein Dijkstra* \\ Debye Institute for Nanomaterials Science, Utrecht University, Princetonplein 5, 3584CC Utrecht, The Netherlands
}

\section{Supporting Information}

ABSTRACT: Colloidal photonic crystals display peculiar optical properties that make them particularly suitable for application in different fields. However, the low packing fraction of the targeted structures usually poses a real challenge in the fabrication stage. Here, we propose a route to colloidal photonic crystals via a binary mixture of hard tetramers and hard spheres. By combining theory and computer simulations, we calculate the phase diagram as well as the stacking diagram of the mixture and show that a colloidal analogue of the $\mathrm{MgCu}_{2}$ Laves phase-which can serve as a precursor of a photonic band-gap structure-is a thermodynamically stable phase in a large region of the phase diagram. Our findings show a relatively large coexistence region between the fluid and the Laves phase, which is potentially accessible by experiments. Furthermore, we determine the sedimentation behavior of the suggested mixture, by identifying several stacking sequences in the sediment. Our work uncovers a self-assembly path toward a photonic structure with a band gap in the visible region.

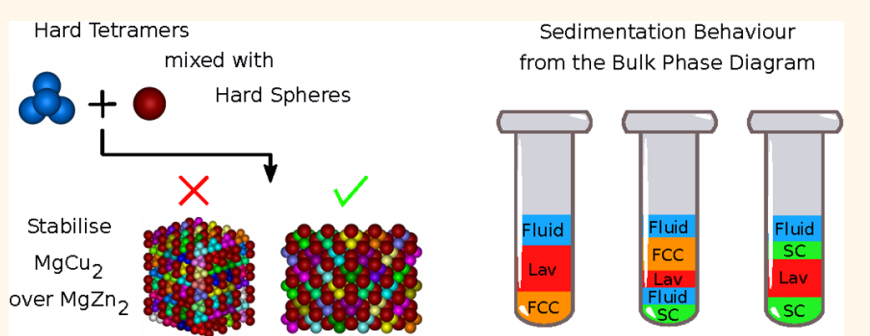

KEYWORDS: colloidal particles, Laves phases, hard tetramers, Monte Carlo methods, phase diagrams, sedimentation, photonic crystals

I $\mathrm{t}$ is known that colloidal particles can spontaneously form ordered, periodic phases that are the analogue of crystals in atomic systems. The most prominent example of such a transition, first discovered by computer simulations ${ }^{1}$ and later confirmed by experimental work, ${ }^{2}$ is the formation of a facecentered-cubic (FCC) crystal from a fluid of colloidal particles that behave as hard spheres.

The study of crystalline phases on colloidal length and time scales is important not only at a fundamental level, where it allows for insights into, for example, phase transitions and crystallization kinetics, ${ }^{3,4}$ but also for potential applications. In particular, it is possible to fabricate from colloidal particles photonic crystals, which are structures with a periodically varying dielectric constant that display a complete photonic band gap. Due to the intrinsic size of the employed building blocks, colloidal photonic crystals display a band gap in the visible range of frequencies. These structures act for photons in the same way as semiconductors do for electrons, hence opening up a way to control light propagation. The applicability of such materials is quite broad, ranging from optical fibers, displays and switches to (bio)sensing and biomedical engineering, and finally to energy storage and security. ${ }^{5-8}$ Therefore, a significant amount of research in the colloid science community deals with the design and fabrication of such photonic crystals.

Since the early work on photonic crystals, ${ }^{9,10}$ different particle arrangements were explored as candidates, ${ }^{11,12}$ and some of them-most notably the so-called "inverse opals"were also fabricated in the lab. ${ }^{13,14}$ To date, the most suitable candidates to make photonic crystals remain the diamond crystal and the pyrochlore structure, in which the colloids are located on the lattice positions of the respective crystal structures. ${ }^{15,16}$ However, despite the efforts, the fabrication of such open (non-close-packed) structures at the colloidal scales has not been achieved yet, and it is a long-standing research focus in the nanomaterials and colloid science community.

Nevertheless, new perspectives on the subject arise because the recent advances in colloid synthesis allow for more and more exotic building blocks to be used in the colloidal selfassembly arena. Clusters of spheres with well-defined shapes, such as dimers, trimers, and tetramers, have become available, together with the intriguing possibility of employing them to self-assemble into photonic crystals. ${ }^{17-20}$ These colloidal clusters can be produced in several ways. One method takes advantage of the drying forces in an evaporating emulsion droplet to drive the confined colloidal particles to a specific geometry. ${ }^{17,18}$ A different class of fabrication procedures relies

Received: January 23, 2017

Accepted: July 31, 2017

Published: August 8, 2017 
instead on microfluidics setups, with or without the use of lithographically patterned surfaces. ${ }^{19}$

In addition, on the theoretical side, two new ideas were put forward to facilitate the fabrication of photonic crystals, and we shall briefly discuss them in the following. One study showed that a structure composed of tetrahedral clusters of spheres ("tetrastack") displays a photonic band gap in the optical region. ${ }^{21}$ However, while they employ a complex building block, it is not clear how the suggested structure can be realized experimentally. Another study suggested that, by using a binary mixture of colloidal particles with different sizes, it is possible to assemble a $\mathrm{MgCu}_{2}$ Laves phase. This is appealing because the $\mathrm{MgCu}_{2}$ consists of a diamond crystal of large spheres and a pyrochlore lattice of small spheres, and both substructures display a photonic band gap. ${ }^{22}$ In this case, the authors addressed the problem posed by the open structure by using a binary mixture of spheres. Nevertheless, issues arise when one considers that three phases can actually be assembled from a binary hard-sphere mixture, namely, the $\mathrm{MgCu}_{2}$, the $\mathrm{MgNi}_{2}$, and the $\mathrm{MgZn}_{2}$. It is also important to note that the latter is the thermodynamically stable phase and unfortunately not the aimed-for $\mathrm{MgCu}_{2}$ phase. ${ }^{23}$ Furthermore, the three aforementioned Laves phases are nearly degenerate, as they have very similar free energies; hence the self-assembly of the mixture results in glassy states, unless the assembly is directed, for example, by using templated walls. ${ }^{22}$

In this work, we combine Monte Carlo (MC) computer simulations and theoretical calculations to study the phase behavior of a binary mixture of large hard spheres and rigid, hard tetrahedral clusters of small hard spheres (hereafter denoted as tetramers) with a fixed size ratio. For this mixture, we compute both the bulk phase diagram and the sedimentation behavior. In particular, using free-energy calculations, we address the stability of the $\mathrm{MgCu}_{2}$ Laves phase that can result from the self-assembly of the mixture. In this way, we retain the best of both approaches previously introduced, while also circumventing some of the other problems. For instance, employing a binary mixture mitigates the problem of the low-coordinated open target structures of the diamond and pyrochlore phase, whereas using tetramers as one of the building blocks alleviates the lattice degeneracy problem, as the $\mathrm{MgNi}_{2}$ phase cannot be self-assembled from tetramers and spheres. Moreover, using tetramers allows one to remove the metastability problem since in this case the $\mathrm{MgCu}_{2}$ phase becomes more stable than the $\mathrm{MgZn}_{2}$, as we shall show. Finally, by using the bulk phase diagram and the local density approximation, we theoretically calculate the stacking diagram of the mixture, which predicts the stacking sequences of different phases that could be observed in sedimentation experiments on the same mixture.

We stress that such a model mixture is well within experimental reach, even though no studies on it have been performed yet, to the best of our knowledge. This is somewhat surprising, as hard-core systems are usually much easier to control than systems with attractive interactions, which often requires substantial fine-tuning of the range, strength, and directionality of the interactions. However, it is important to mention here that very recently the $\mathrm{MgCu}_{2}$ Laves phase was found experimentally by using colloidal spheres and preassembled tetrahedral clusters of spheres coated by DNA in order to induce short-range attractive interactions between the unlike species. $^{24}$

\section{RESULTS AND DISCUSSION}

System and Model. Monte Carlo Simulations. We consider a binary mixture of $N_{\mathrm{s}}$ hard spheres and $N_{\mathrm{t}}$ hard tetramers with composition $x=N_{\mathrm{s}} / N$, where $N=N_{\mathrm{s}}+N_{\mathrm{t}}$. The spheres have diameter $\sigma_{\mathrm{L}}$. Each tetramer consists of four touching spherical beads of diameter $\sigma_{\mathrm{B}}$ arranged in a tetrahedral fashion. We assume that the tetramers behave like a rigid body; i.e., fluctuations in the geometrical arrangement of the spheres are neglected. The size ratio between a bead in a tetramer and a sphere is labeled as $q=\sigma_{\mathrm{B}} / \sigma_{\mathrm{L}}$. Since the $\mathrm{MgCu}_{2}$ Laves phase of an ordinary binary hard-sphere mixture achieves its highest packing fraction for $q=\sqrt{2 / 3} \approx 0.82,22,23,25,26$ we employ this value in our work. All interactions are assumed to be hard-sphere-like, meaning that the objects do not interpenetrate each other. Thus, spheres cannot approach each other closer than $\sigma_{\mathrm{L}}$, beads belonging to different tetramers cannot approach each other closer than $\sigma_{\mathrm{B}}$, spheres and tetramer beads cannot approach closer than $\sigma_{\mathrm{LB}}=\left(\sigma_{\mathrm{L}}+\sigma_{\mathrm{B}}\right) / 2$. The pressure $P$ is measured in reduced units as $\beta P \sigma_{\mathrm{L}}^{3}$ where $\beta$ $=1 / k_{\mathrm{B}} T, k_{\mathrm{B}}$ is Boltzmann's constant, and $T$ is the (irrelevant) system temperature. The packing fraction is defined as $\eta=\gamma \rho$, where $\rho=N / V$ is the number density, $V$ is the volume of the simulation box, and $\gamma=\pi \sigma_{\mathrm{L}}{ }^{3}\left[x+4 q^{3}(1-x)\right] / 6$. The Gibbs free energy per particle is defined as $g=\beta G / N=f+\gamma \beta P / \eta$, where $f$ $=\beta \mathrm{F} / N$ is the dimensionless Helmholtz free energy. More details on the simulations and the free-energy calculations can be found in the SI. A model of the different building blocks employed in this work is shown in Figure 1.

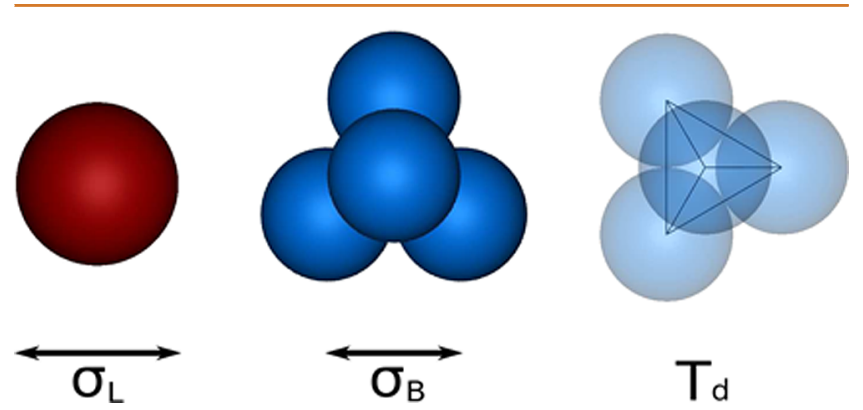

Figure 1. Building blocks of the investigated binary mixture. (Left) Hard spheres with diameter $\sigma_{\mathrm{L}}$. (Center) Hard tetrahedral clusters of spherical beads with size $\sigma_{\mathrm{B}}$. Note that the beads are tangential to one another. The size ratio $q=\sigma_{\mathrm{B}} / \sigma_{\mathrm{L}}$ is fixed to 0.82 . (Right) Faceted model of a tetrahedron, with symmetry group $T_{d}$, connecting the centers of the beads.

Crystalline Structures. For a binary hard-sphere mixture, previous studies have shown that, at the chosen size ratio $q=$ $\sigma_{\mathrm{B}} / \sigma_{\mathrm{L}}=0.82$, the stable crystal structures are the pure FCC crystals of large and of small spheres and the $\mathrm{MgNi}_{2}, \mathrm{MgCu}_{2}$, and $\mathrm{MgZn}_{2}$ Laves phases, ${ }^{22,23}$ where the $\mathrm{MgZn}_{2}$ phase has a slightly lower free energy than the other two. In the case of a mixture of tetramers and spheres, we employ the same packing arrangements as those in ref 23 , but we replace four small spheres by a tetramer. This procedure yields structures that are made from the investigated building blocks (spheres and tetramers), but are arranged similarly to the respective literature cases. In particular, the FCC of small spheres at $x=0$ becomes a simple cubic crystal lattice of tetramers. Furthermore, it is important to note that the third kind of Laves phase-the $\mathrm{MgNi}_{2}$ crystal-cannot be reproduced by a combination of tetramers and spheres; hence it falls already out of the picture 
when considering candidate crystal structures. Summing up, for different compositions of large spheres $x$ we have

- SC, a simple cubic lattice of tetramers with specified orientation at composition $x=0$;

- LP1, a mixed structure of tetramers and spheres, which packs the same way as an ordinary $\mathrm{MgCu}_{2}$ lattice, at composition $x=2 / 3$;

- LP2, the analogue of the $\mathrm{MgZn}_{2}$ crystal, but made out of tetramers and spheres, also at composition $x=2 / 3$. Note that this structure has a noncubic unit cell;

- FCC, the thermodynamic stable structure for hard spheres, at composition $x=1$.

In the SC, LP1, and LP2 phases, respectively, all the tetramers initially have the same orientation, which is calculated by a rigid transformation of the bead positions in the reference frame of the tetramers to the bead positions in the crystal at hand. The SC phase can also be formed by stacking different layers of tetramers on top of each other, in which the tetramers are either shifted or rotated, nevertheless the positions of the beads of the tetramers must always be compatible with an FCC packing. The different crystal structures of the SC phase, which have been considered in detail elsewhere, ${ }^{27}$ have very similar free energies and do not affect the main results presented here concerning the Laves phases nor the general topology of the phase diagram and were therefore neglected. We also note here that we exclude the possibility of an aperiodic crystal, in which the tetramers are randomly oriented.

Stacking Sequences and Stacking Diagram. Once the bulk equilibrium phase diagram is ascertained, we also study the sedimentation behavior of the system. To this end, we theoretically construct a stacking diagram that gives all possible stacking sequences of phases in a sedimentation-diffusion equilibrium, following the method recently presented in ref 28 . The theory behind the construction of a stacking diagram is based on chemical potentials, hence the bulk phase diagram in the $P-x$ representation must first be converted to the plane of chemical potential of the spheres (L) and tetramers $(\mathrm{T})$, respectively. In the following, we assume that such a conversion has been done, and we discuss the method to determine the stacking diagram only in terms of chemical potentials of the two species.

Once gravity is switched on, an inhomogeneous sedimentation-diffusion equilibrium profile is obtained in the direction of gravity $z$. Employing the local density approximation (LDA), we define a local chemical potential $\mu_{i}(z)$ of species $i=\mathrm{L}, \mathrm{T}$, which depends linearly on the height $z$ in the sedimentationdiffuion equilibrium:

$$
\mu_{i}(z)=\mu_{i}^{0}-m_{i} g z
$$

where $\mu_{i}^{0}$ is the total chemical potential of species $i$ in the absence of gravity in the system and $m_{i}$ is the buoyant mass of species $i$. Rearranging eq 1 and eliminating the $z$-dependence, we obtain a linear relation between the chemical potential of the spheres $\mu_{\mathrm{L}}(z)$ and the chemical potential of the tetramers $\mu_{\mathrm{T}}(z)$

$$
\mu_{\mathrm{L}}\left(\mu_{\mathrm{T}}\right)=a+s \mu_{\mathrm{T}}
$$

where the slope $s=m_{\mathrm{L}} / m_{\mathrm{T}}$ is the gravitational variable, i.e., the buoyant mass ratio of spheres and tetramers, and $a=\mu_{\mathrm{L}}^{0}-s \mu_{\mathrm{T}}^{0}$ is the composition variable. For a given $a$ and s, i.e., fixed overall composition and buoyant masses of the two species, eq 2 represents a straight line in the phase diagram in the $\mu_{\mathrm{T}}-\mu_{\mathrm{L}}$
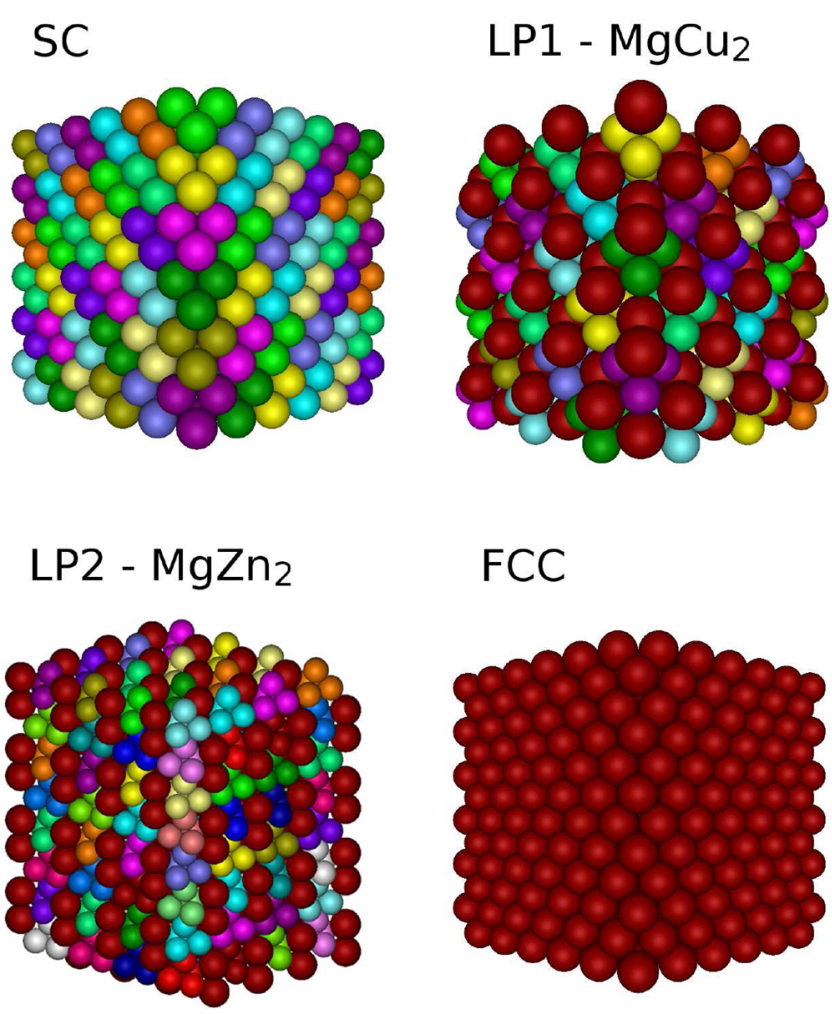

Figure 2. Crystal structures considered in this work. (Top left) The simple cubic crystal phase of hard tetramers (SC) at composition $x$ = 0. (Top right) The binary $\mathrm{MgCu}_{2}$ Laves phase (LP1) at composition $x=2 / 3$. (Bottom left) The binary $\operatorname{MgZn}_{2}$ Laves phase (LP2) at composition $x=2 / 3$. (Bottom right) The face centered cubic crystals of large spheres (FCC) at composition $x=1$. The color code identifies different tetramers and separates tetramers from spheres.

representation. This straight line is called a "sedimentation path". The point at which a sedimentation path crosses a bulk binodal represents an interface between two phases in the sedimentation column. Therefore, each sedimentation path in the $\mu_{\mathrm{T}}-\mu_{\mathrm{L}}$ phase diagram corresponds to a specific stacking sequence of phases in the sedimentation column. Thus, the stacking sequence is determined by the slope $s$, i.e., the ratio of the buoyant masses of the two species, the direction of the sedimentation path denoted by the sign of $s$, the overall composition and concentration given by $a$, and the height of the sample. All possible stacking sequences are summarized in a so-called stacking diagram.

Equations of State. The equations of state (EOS) of both the fluid phase at different compositions $x$ and of the crystalline structures considered are a key ingredient of the calculation of the phase diagram. For the fluid phase, we calculated the EOSs at composition intervals of 0.1 , whereas for the crystal phases the compositions are fixed. In Figure 3 we show the EOSs of the different crystal structures investigated, as well as the EOSs of the fluid mixture at different compositions $x$. We fit the simulation results to

$$
\frac{\gamma \beta P}{\eta}=1+\sum_{i=1}^{n} a_{i} \eta^{i}
$$

for the fluid phase, and 


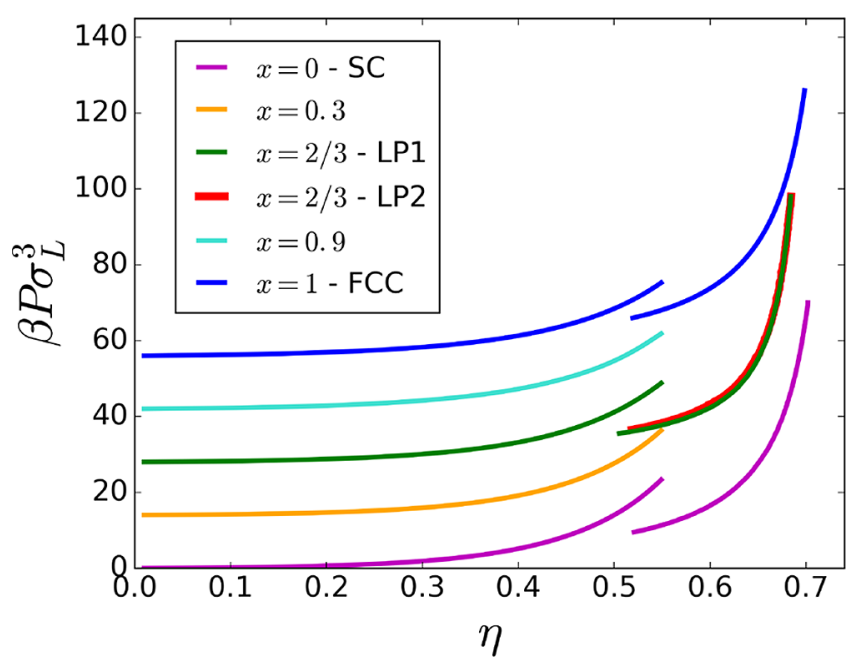

Figure 3. Pressure $\beta P \sigma_{\mathrm{L}}^{3}$ as a function of packing fraction $\eta$ (EOS) for a mixture of large hard spheres and hard tetramers at different sphere compositions $x=N_{S} / N$. The branches at high $\eta$ correspond to the studied solid structures, namely, SC at $x=0, \mathrm{LP1}-\mathrm{MgCu}_{2}$ and LP2-MgZn ${ }_{2}$ at $x=2 / 3$, and FCC at $x=1$. Note that the EOSs of LP1 and LP2 coincide for high pressures, but differ at lower pressures. For visualization purposes, the curves have been shifted with respect to each other in the $y$ direction by an amount $\Delta y=14$.

$$
\frac{\gamma \beta P}{\eta^{2}}=\sum_{i=0}^{m} b_{i} \eta^{i}
$$

for the crystal phases. The typical value of $n$ is 12 , while $m=3$ for all cases. The fitting procedure allows us to easily perform the thermodynamic integration as described in the SI.

Stability of $\mathrm{LP1}-\mathrm{MgCu}_{2}$ and Phase Diagrams. Previous work on binary hard-sphere mixtures has shown that the $\mathrm{MgZn}_{2}$ Laves phase is more stable than the $\mathrm{MgCu}_{2}$ Laves phase. ${ }^{2,23}$ Unfortunately, the $\mathrm{MgCu}_{2}$ structure is the only Laves phase whose sublattices display a complete photonic band gap. ${ }^{15,16}$ Hence, the first issue for us to investigate is the thermodynamic stability of $\mathrm{LP} 1-\mathrm{MgCu}_{2}$ compared to LP2$\mathrm{MgZn}_{2}$. We addressed this by performing free-energy calculations at a fixed packing fraction of $\eta=0.60$ for different total number of particles $N$. By plotting the excess free energy per particle including finite-size corrections versus $1 / N$ for both structures, we can extrapolate to the thermodynamic limit $N \rightarrow$ $\infty$ by looking at the intercept of the two lines. This is displayed in Figure 4, where it becomes clear that the $\mathrm{LP1}-\mathrm{MgCu}_{2}$ structure of hard tetramers and hard spheres is more stable than the $\mathrm{LP} 2-\mathrm{MgZn}_{2}$ structure in the thermodynamic limit. The LP1 structure has a bulk excess free energy per particle of $10.01(1) k_{\mathrm{B}} T$, while the LP2 crystal has an excess free energy per particle of $10.07(1) k_{\mathrm{B}} T$, the difference being $6 \times 10^{-2} k_{\mathrm{B}} T$. Incidentally, we note that this free-energy difference is not at all small, being tens of times larger than the free-energy difference between a face-centered-cubic and an hexagonal close-packing of hard spheres. Thus, by employing a mixture of hard tetramers and large hard spheres, the $\mathrm{MgCu}_{2}$ structure-the precursor of colloidal photonic crystals-is stabilized in the bulk. In view of this result, we will refer to LP1 more generically as the "Laves phase" in the following.

To draw the phase diagram in the pressure $\beta P \sigma_{\mathrm{L}}^{3}-$ composition $x$ representation, we apply common tangent constructions to the Gibbs free-energy curves $g(P, x)$ at different pressures, in order to determine the composition and

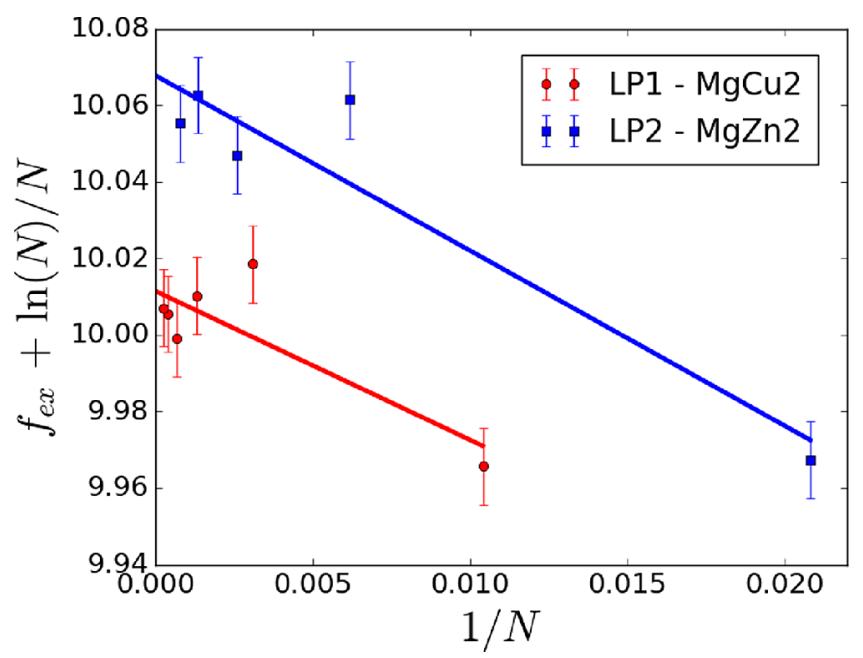

Figure 4. Finite-size scaling of the excess Helmholtz free energy $f_{\text {ex }}$ $+\ln (N) / N$ vs $1 / N$ at diameter ratio $q=0.82$ and packing fraction $\eta$ $=0.6$ for the $\mathrm{LP1}-\mathrm{MgCu}_{2}$ and $\mathrm{LP2}-\mathrm{MgZn}_{2}$ Laves structures of hard tetramers and hard spheres. The lines are linear fits to the data points. The LP1 crystal is always significantly more stable than the LP2 structure, the free energy difference in the thermodynamic limit being $6 \times 10^{-2} k_{\mathrm{B}} T$ per particle.

densities of the coexisting phases. The results are summarized in Figure 5.

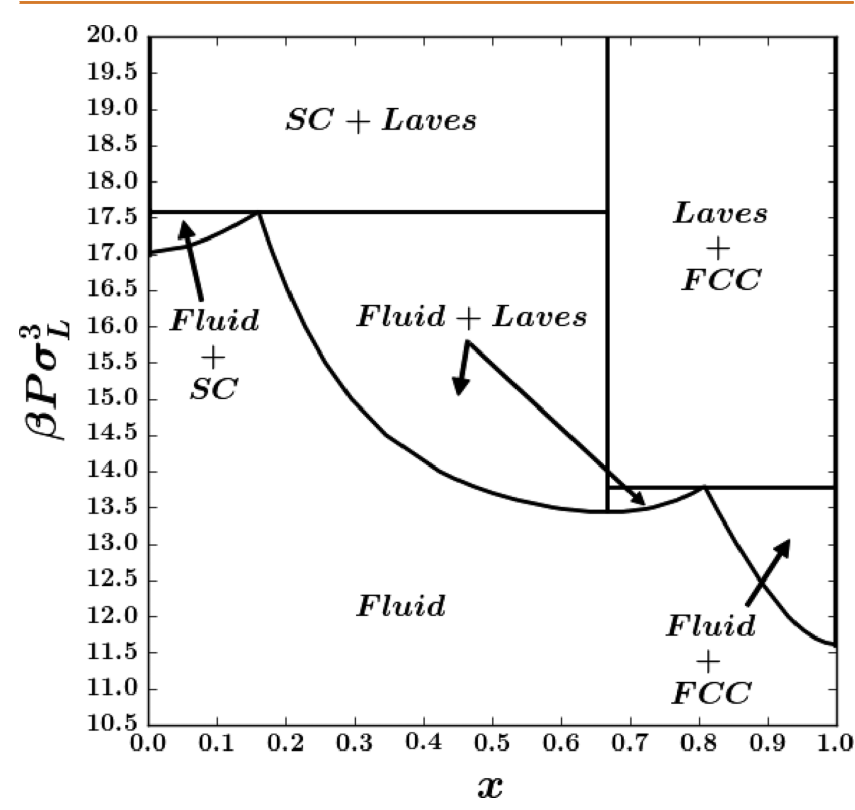

Figure 5. Phase diagram of a binary mixture of hard spheres and hard tetramers in the pressure $\beta P \sigma_{\mathrm{L}}^{3}$-composition $x$ representation. The composition $x=N_{\mathrm{S}} / N$ refers to the spheres. Two triple points (Fluid+SC+Laves, Fluid+Laves+FCC) are found, together with a relatively large phase coexistence region between the fluid and the Laves phase.

For pressures $\beta P \sigma_{\mathrm{L}}^{3} \leq 11.5$, we find that the fluid is the only stable phase. Increasing the pressure results in different coexistence regions, between the fluid and the three crystal structures investigated, and between the different crystal structures at even higher pressures.

For $11.5 \leq \beta P \sigma_{\mathrm{L}}^{3} \leq 13.9$ and compositions $x>0.81$ we find phase coexistence between the FCC crystal of large spheres and 
the fluid phase, while for $17.0 \leq \beta P \sigma_{\mathrm{L}}^{3} \leq 17.6$ and compositions $x<0.17$ we find a coexistence between the simple cubic crystal of tetramers and the fluid phase.

Interestingly, at intermediate pressures and compositions we observe two distinct phase coexistence regions between the Laves phase and the fluid phase with either a composition smaller or larger than that of the Laves phase, i.e., $x \leq 2 / 3$ and $x$ $\geq 2 / 3$. Moving toward high pressures we find solid-solid coexistence between the simple cubic phase of pure tetramers and the Laves phase and between the Laves phase and the pure FCC phase of large spheres, the former starting at somewhat higher pressures than the latter $\left(\beta P \sigma_{\mathrm{L}}^{3}>17.6 v s \beta P \sigma_{\mathrm{L}}^{3}>13.9\right)$.

For very high pressures, we expect, due to packing considerations, only a single coexistence region between the simple cubic phase of tetramers and the FCC crystals of large spheres; i.e., we expect to find another triple point where the SC, Laves, and FCC phases are in coexistence with each other. However, we were unable to detect the crossover, even by simulating at pressures as high as $\beta P \sigma_{\mathrm{L}}^{3}=70.0$. Thus, we can only set a lower limit on this specific crystal-crystal phase coexistence region.

The relatively large two-phase coexistence region between the fluid phase and the Laves phase is the most remarkable feature of the presented phase diagram, signaling an extended and easily accessible parameter range to obtain the targeted $\mathrm{MgCu}_{2}$ Laves phase in simulations as well as in experiments. We checked the mechanical stability of the phase coexistence between the fluid and the Laves phase by performing direct coexistence simulations at overall compositions $x=0.5$ and $x=$ 0.6 and pressure $\beta P \sigma_{\mathrm{L}}^{3}=15.0$. In Figure 6 we present snapshots

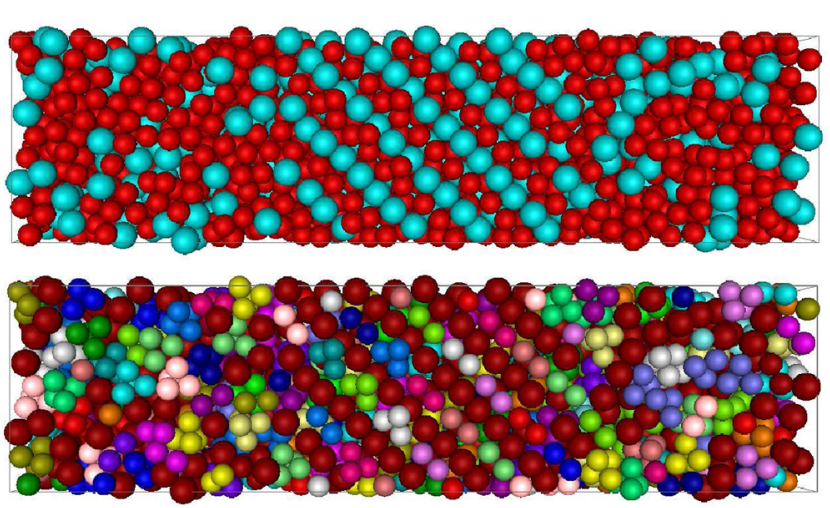

Figure 6. Representative final configuration from direct coexistence simulations displaying coexistence between the fluid phase and the Laves phase of hard tetramers and hard spheres. (Top) Overall composition $x=0.6$ and pressure $\beta P \sigma_{\mathrm{L}}^{3}=15.0$. (Bottom) Same as top panel, but with color coding to highlight the different tetramers.

of the final configurations as obtained from the simulations, which confirm the coexistence between the fluid phase and the Laves phase of tetramers and spheres.

Despite the progress in the fabrication of colloidal building blocks, we are unaware, to the best of our knowledge, of experimental realizations of the proposed binary mixture. In order to facilitate the comparison with experimental results, we additionally convert the phase diagram to the packing fraction of tetramers $\eta_{\mathrm{T}}$-packing fraction of spheres $\eta_{\mathrm{S}}$ representation, the result being shown in Figure 7. The triple points we found in Figure 5-Fluid + SC + Laves, Fluid + Laves + FCCtransform to triangular areas in this representation. In between

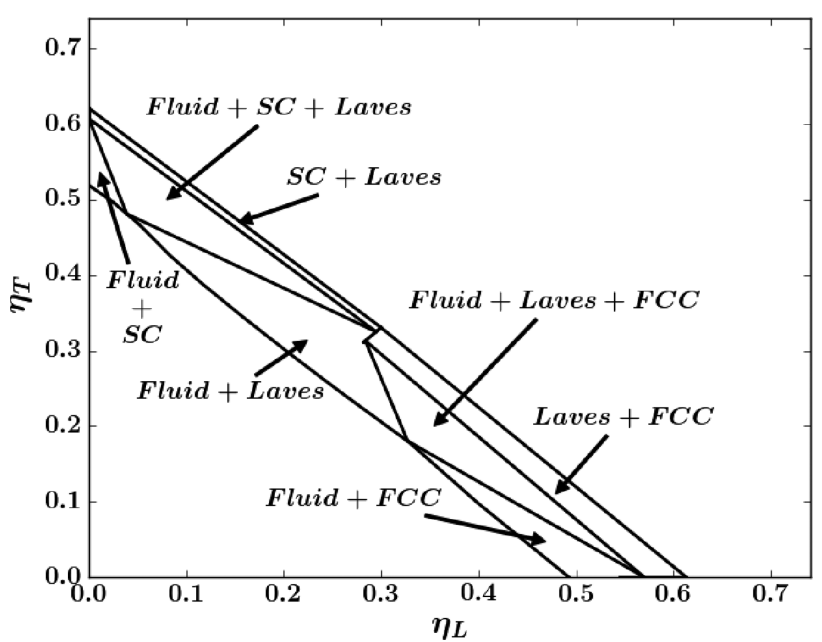

Figure 7. Phase diagram of the investigated binary mixture in the packing fraction of tetramers $\eta_{\mathrm{T}}$-packing fraction of large spheres $\eta_{\mathrm{L}}$ representation.

the triple points we find the coexistence region between fluid phase and Laves structure, which could be probed experimentally. Finally, we also calculate the phase diagram in the chemical potential of the spheres $\mu_{\mathrm{L}}$-chemical potential of the tetramers $\mu_{\mathrm{T}}$ representation. While this diagram is not suitable for comparison with experiments, it is instead crucial in order to theoretically address the role of gravity on the presented bulk results, as accomplished in the next section.

Sedimentation Behavior and Stacking Diagram. We now study the system subject to a gravitational field. The phase diagram in the chemical potential of the spheres $\mu_{\mathrm{L}}$-chemical potential of the tetramers $\mu_{\mathrm{T}}$ representation is shown in Figure 8 , where full black lines represent bulk binodals. At each point on a binodal two phases are in equilibrium with each other.

The bulk phase diagram in the $\mu_{\mathrm{T}}-\mu_{\mathrm{L}}$ plane is used as an input for our theory in order to calculate the stacking diagram in the $a-s$ representation. Exemplarily, we show various sedimentation paths in Figure 8, which are straight lines in the $\mu_{\mathrm{T}}-\mu_{\mathrm{L}}$ phase diagram. The different sedimentation paths correspond to different stacking sequences, and the stacking diagram in the $a-s$ representation summarizes the possible phase sequences. The boundaries between the different regions in the stacking diagram, each of which represents a different stacking sequence, are determined by the following boundary curves.

Sedimentation Binodal. This is the locus of all sedimentation paths tangential to the bulk binodal(s) in the $\mu_{\mathrm{T}}-\mu_{\mathrm{L}}$ phase diagram. We have five bulk binodals in the $\mu_{\mathrm{T}}-\mu_{\mathrm{L}}$ phase diagram indicating the various coexistences as shown in Figure 8 , thus giving five corresponding sedimentation binodals in the stacking diagram.

Terminal Lines. This is the set of all sedimentation paths through an end point of a bulk binodal in the $\mu_{\mathrm{T}}-\mu_{\mathrm{L}}$ phase diagram, i.e., a triple point or a critical point. This end point corresponds to a line in the stacking diagram. To see how this happens, let us label such a point by its coordinates $p=\left(\mu_{\mathrm{T}}^{\text {term }}\right.$, $\left.\mu_{\mathrm{L}}^{\text {term }}\right)$. Plugging this into eq 2 and inverting the resulting relation yields an equation for $a$ as a function of $s$, i.e., $a=a(s)$. This equation represents a so-called "terminal line" on the stacking diagram, and it originates from the terminal point $p$. In the bulk phase diagram of Figure 8 we have three terminal points: 


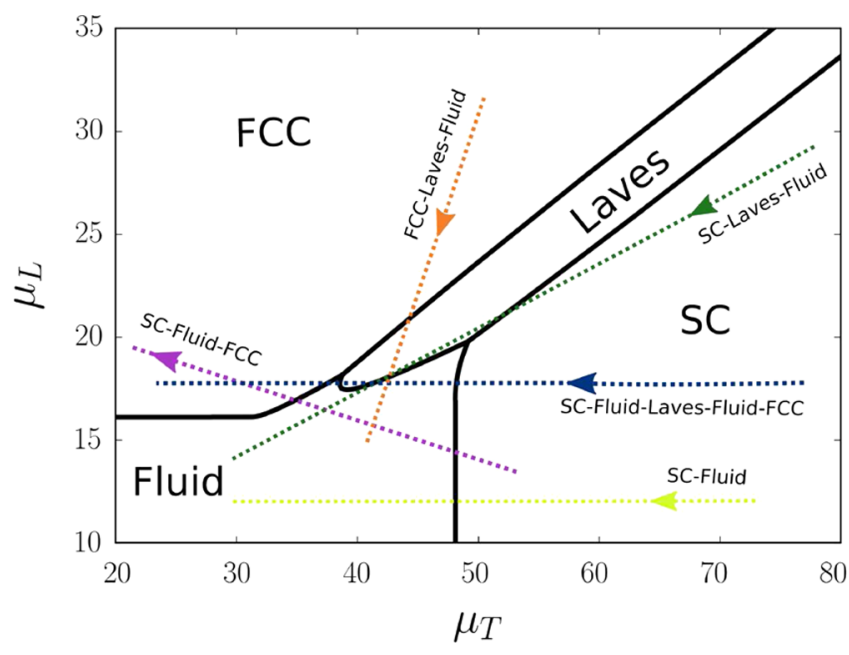

Figure 8. Phase diagram of a binary mixture of hard tetramers and hard spheres in the chemical potential of large hard spheres $\mu_{\mathrm{L}}-$ chemical potential of hard tetramers $\mu_{\mathrm{T}}$ representation. The solid lines represent the bulk binodals and delimit single-phase regions. The phase transitions of the pure system of spheres and the pure system of tetramers are shown by the horizontal and vertical asymptotic extensions of the respective binodals. The colored dashed lines represent some of the possible phase-stacking sequences in the sediment. The color code is the same as the one used for the stacking diagram. The arrows on the dashed lines indicate the direction from the bottom to the top of the sediment.

1. the triple point, visible in Figure 8, where the Laves, fluid, and FCC phases coexist;

2. the triple point, visible in Figure 8, where the Laves, fluid, and SC phases coexist;

3. the triple point, not visible in Figure 8, where the Laves, FCC, and SC phases coexist. In order to locate this point in the plane of the chemical potentials, we obtain the FCC-Laves and SC-Laves binodals from simulations up to pressures $\beta P \sigma_{\mathrm{L}}^{3}=70$, and we extrapolate the last simulated points until the two binodals meet.

Asymptotic Terminal Lines. This is the set of sedimentation paths with the asymptotic slope of a bulk binodal in the $\mu_{\mathrm{T}}-\mu_{\mathrm{L}}$ phase diagram that does not terminate at a finite value of one of the chemical potentials. For example, in our system the fluidFCC binodal terminates in a horizontal asymptote in the $\mu_{\mathrm{T}}-\mu_{\mathrm{L}}$ phase diagram as the chemical potential of the absent species (tetramers) approaches $-\infty$. Here, we remind the reader that the parameter $s$ is the slope of the tangent to the bulk binodal, and in the above-mentioned case $s \rightarrow 0$. Thus, all the possible sedimentation paths parallel to the horizontal asymptotic bulk binodal of large hard spheres result in a vertical line on the stacking diagram located at $s=0$. Similarly, the fluid-SC binodal terminates as a vertical asymptote in the $\mu_{\mathrm{T}}-\mu_{\mathrm{L}}$ phase diagram as the chemical potential of the large hard spheres approaches $-\infty$, and hence this line corresponding to a line at $s=-\infty$ does not appear in the stacking diagram as presented in Figure 9.

By assembling these features together, we obtain the corresponding stacking diagram of a system of large hard spheres and hard tetramers undergoing sedimentation, shown in Figure 9. The differently colored regions in Figure 9 represent the different stacking sequences for this binary mixture. The labels list the different phases in the sediment from bottom to top. We remind the reader that $s=m_{\mathrm{L}} / m_{\mathrm{T}}$ also

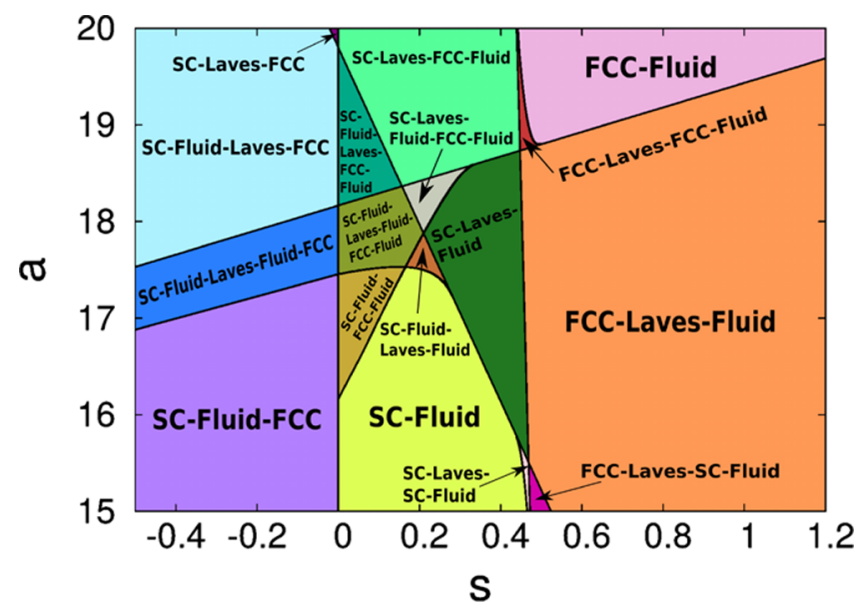

Figure 9. Stacking diagram depicting the sedimentation-diffusion equilibrium for the binary mixture of large hard sphere-hard tetramer. The colored regions represent the different phasestacking sequences of phases observed in the sedimentation column. The labels list the different phases in the sediment from bottom to top. Note that the colors of the regions correspond to the colors of the sedimentation paths drawn on the phase diagram in the $\mu_{\mathrm{T}}-\mu_{\mathrm{L}}$ representation in Figure 8. For visualization purposes we have restricted the axes to the region that contains the largest amount of stacking sequences. Moreover, the $a$ axis was linearly scaled with respect to $s$ by a constant $c=-40$, such that $a=a_{\text {actual }}-$ cs.

equals the ratio of the buoyant masses of the spheres to the tetramers, see eq 2 . A negative $s$ means that one species settles while the other floats up. In the present work, we assume that the tetramers always settle, which means that the buoyant mass of the tetramers is always positive, i.e., $m_{\mathrm{T}}>0$. Alternatively, if the identity of the settling species is switched, the stacking sequences for the negative $s$ region will simply be reversed. Keeping this in mind, the following observations can be made about the resulting stacking diagram.

1. For negative $s$, the single-species crystal phase formed at the bottom is always the SC phase of tetramers, as is expected. At the top of the sediment, we find a singlespecies FCC phase, which can be explained by the values for $a$ corresponding to the overall concentrations and compositions of the system as considered in the present stacking diagram.

2. For $s>0$, we find always a fluid phase at the top of the sediment for the values of $a$ considered here.

3. For $s>1$, the FCC crystal phase of large spheres is formed at the bottom, as the spheres have a higher buoyant mass than the tetramers.

4. The regime $0.45 \lesssim s=m_{\mathrm{L}} / m_{\mathrm{T}} \lesssim 1$ is interesting because, although the tetramers are heavier, the FCC crystal phase of large spheres is predicted to form at the base of the column, which may seem counterintuitive. We explain this result in terms of the total chemical potential difference between the large spheres $\mu_{\mathrm{L}}^{0}$ and the tetramers $\mu_{\mathrm{T}}^{0}$, which favors the FCC crystal at the bottom of the sediment.

5. We found some counterintuitive stacking sequences with up to six different phases in a sediment, such as SCFluid-Laves-Fluid-FCC-Fluid, as well as floating phases with crystalline phases sandwiched between two 
fluid phases, ${ }^{29}$ such as SC-Fluid-Laves-Fluid or SCFluid-FCC-Fluid phase sequences.

\section{CONCLUSIONS}

We investigated the phase behavior of a binary mixture of hard spheres and hard tetramers consisting of beads arranged in a tetrahedral fashion. By using MC simulations in the isobaricisothermal ensemble combined with free-energy calculations and the thermodynamic integration method, we mapped out the bulk phase diagram of the mixture in the pressure $\beta P \sigma_{\mathrm{L}}^{3}-$ composition $x$ representation.

We found phase coexistence regions between the fluid phase and various crystal structures, as well as two triple points, namely, the Fluid+SC+Laves and the Fluid+Laves+FCC triple points. Surprisingly, we find a relatively large coexistence region between the fluid and the Laves phase, the structural analogue of the $\mathrm{MgCu}_{2}$ phase, which may be experimentally accessible. In order to facilitate comparison with experimental parameters, we also converted the phase diagram from the pressure $\beta \mathrm{P} \sigma_{\mathrm{L}}^{3}-$ composition $x$ representation to the packing fraction of tetramers $\eta_{\mathrm{T}}$-packing fraction of spheres $\eta_{\mathrm{L}}$ plane.

Assuming the validity of the local density approximation under relevant experimental conditions for our binary system, we also investigated the sedimentation behavior by calculating the stacking diagram of this mixture. Some of the stacking sequences are highly nontrivial, displaying, for example, floating crystal phases.

Our results demonstrate a self-assembly route toward a photonic material where the diamond and the pyrochlore structures can be assembled in one crystal-the $\mathrm{MgCu}_{2}$ Laves structure-from a binary mixture of hard spheres and hard tetramers. By selectively burning or dissolving one of the species, either the tetramers or the spheres, the Laves phase can be converted into a diamond lattice or a pyrochlore structure to obtain a photonic crystal with a band gap in the visible range. We thus showed that the $\mathrm{MgCu}_{2}$ Laves phase is thermodynamically stable in the phase diagram of a binary mixture of hard tetramers and hard spheres.

However, it is important to remark here that besides the thermodynamic stability of the Laves phase as investigated here, kinetics also plays an important role in the self-assembly of the Laves phase. For instance, the self-assembly of the Laves phase can be hampered by kinetic limitations such as vitrification, kinetic trapping in metastable states, or slow dynamics. In addition, the kinetic pathways for crystallization may be influenced by hydrodynamics or changes in the interaction potentials. For instance, it has been recently shown that DNA coating facilitates the crystallization of the Laves phases. ${ }^{24}$ The crystallization kinetics and the different pathways for nucleation will be left for future studies. With an outlook on the experimental feasibility, we also surmise that the polydispersity in the tetramer beads and in the tetramer overall aspect ratio would play a role in the self-assembly process. Additionally, while the chosen size ratio maximizes the volume fraction of the Laves phase at close-packing, other size ratios could be investigated that lower the nucleation barrier of the Laves phase. Studying the effect of colloidal epitaxy on this system would be another interesting research direction.

\section{METHODS}

In order to map out the phase diagram of the system, we combine Monte Carlo simulations in the isobaric-isothermal ensemble and free-energy calculations. Hence, the relevant thermodynamic quantities are $N_{\mathrm{s}}, N_{\mathrm{t}}, P$, and $T$. The pressure $P$ is measured in reduced units as $\beta P \sigma_{\mathrm{L}}^{3}$ where $\beta=1 / k_{\mathrm{B}} T, k_{\mathrm{B}}$ is Boltzmann's constant, and $T$ is the system temperature. The packing fraction is defined as $\eta=\gamma \rho$, where $\rho=N / V$ is the number density, $V$ is the volume of the simulation box, and $\gamma=$ $\pi \sigma_{\mathrm{L}}^{3}\left[x+4 q^{3}(1-x)\right] / 6$. To evolve the system, we use displacement moves for spheres and tetramers, rotational moves for tetramers, and volume moves. For each move, we set an acceptance rate of $30 \%$. An MC step (MCS) is defined as $N$ attempted translations or rotations and one volume move of the simulation box. The length of the simulations in the isobaric-isothermal ensemble is at least $5 \times 10^{6}$ MCS, while the free-energy calculations run for at least $2 \times 10^{6}$ MCS. For the case of noncubic crystal structures, we also employ NPT simulations where the box lattice vectors are free to fluctuate, in order to remove any additional stress from the crystal phase. ${ }^{30,31}$ For each composition of large spheres $x$, the equation of state is computed by means of compression and expansion runs. For the compression runs, the starting configuration is a disordered fluid of $N_{\mathrm{s}}=x \mathrm{~N}$ spheres and $N_{\mathrm{t}}=(1-x) N$ tetramers. For the expansion runs, crystalline structures of selected composition provide the initial configuration as explained in the main text. The details on the free-energy calculations can be found in the SI.

\section{ASSOCIATED CONTENT}

\section{Supporting Information}

The Supporting Information is available free of charge on the ACS Publications website at DOI: 10.1021/acsnano.7b00505.

Details on the free-energy calculations used for this work (PDF)

\section{AUTHOR INFORMATION}

\section{Corresponding Author}

*E-mail: m.dijkstra@uu.nl.

ORCID $\odot$

Guido Avvisati: 0000-0002-1012-4651

\section{Author Contributions}

G.A. calculated the EOS of the system, performed the freeenergy calculations, and constructed the phase diagrams of the system. T.D. calculated the stacking diagram of the system. M.D. coordinated the study and helped in writing the manuscript.

\section{Notes}

The authors declare no competing financial interest.

\section{ACKNOWLEDGMENTS}

This work is part of the research program of the Foundation for Fundamental Research on Matter (FOM), which is part of The Netherlands Organisation for Scientific Research (NWO). T.D. acknowledges funding from the Industrial Partnership Programme (Grant No. 13CSER025), which is cofinanced by FOM and Shell Global Solutions International B.V. G.A. thanks L. Filion and S. Dussi for fruitful discussions. The authors thank H. Pattabhiraman and V. Prymidis for critically reading the manuscript.

\section{REFERENCES}

(1) Alder, B. J.; Wainwright, T. E. Phase Transition for a Hard Sphere System. J. Chem. Phys. 1957, 27, 1208-1209.

(2) Pusey, P. N.; Van Megen, W. Phase Behaviour of Concentrated Suspensions of Nearly Hard Colloidal Spheres. Nature 1986, 320, $340-342$.

(3) Anderson, V. J.; Lekkerkerker, H. N. W. Insights into Phase Transition Kinetics from Colloid Science. Nature 2002, 416, 811-815.

(4) Li, B.; Zhou, D.; Han, Y. Assembly and Phase Transitions of Colloidal Crystals. Nature Reviews Materials 2016, 1, 15011. 
(5) Stein, A.; Wilson, B. E.; Rudisill, S. G. Design and Functionality of Colloidal-Crystal-Templated Materials - Chemical Applications of Inverse Opals. Chem. Soc. Rev. 2013, 42, 2763-2803.

(6) Cong, H.; Yu, B.; Tang, J.; Li, Z.; Liu, X. Current Status and Future Developments in Preparation and Application of Colloidal Crystals. Chem. Soc. Rev. 2013, 42, 7774-7800.

(7) Xu, J.; Guo, Z. Biomimetic Photonic Materials with Tunable Structural Colors. J. Colloid Interface Sci. 2013, 406, 1-17.

(8) Ge, J.; Yin, Y. Responsive Photonic Crystals. Angew. Chem., Int. Ed. 2011, 50, 1492-1522.

(9) Bykov, V. P. Spontaneous Emission from a Medium with a Band Spectrum. Quantum Electron. 1975, 4, 861.

(10) Yablonovitch, E. Inhibited Spontaneous Emission in Solid-State Physics and Electronics. Phys. Rev. Lett. 1987, 58, 2059-2062.

(11) Ho, K. M.; Chan, C. T.; Soukoulis, C. M. Existence of a Photonic Gap in Periodic Dielectric Structures. Phys. Rev. Lett. 1990, $65,3152-3155$.

(12) Sözüer, H.; Dowling, J. P. Photonic Band Calculations for Woodpile Structures. J. Mod. Opt. 1994, 41, 231-239.

(13) Lin, S.-Y.; Fleming, J.; Hetherington, D.; Smith, B.; Biswas, R.; Ho, K.; Sigalas, M.; Zubrzycki, W.; Kurtz, S.; Bur, J. A ThreeDimensional Photonic Crystal Operating at Infrared Wavelengths. Nature 1998, 394, 251-253.

(14) Vlasov, Y. A.; Bo, X.-Z.; Sturm, J. C.; Norris, D. J. On-Chip Natural Assembly of Silicon Photonic Bandgap Crystals. Nature 2001, 414, 289-293.

(15) Maldovan, M.; Thomas, E. L. Diamond-Structured Photonic Crystals. Nat. Mater. 2004, 3, 593-600.

(16) Vermolen, E. C. M.; Thijssen, J. H. J.; Moroz, A.; Megens, M.; van Blaaderen, A. Comparing photonic band structure calculation methods for diamond and pyrochlore crystals. Opt. Express 2009, 17, 6952-6961.

(17) Manoharan, V. N.; Elsesser, M. T.; Pine, D. J. Dense Packing and Symmetry in Small Clusters of Microspheres. Science 2003, 301, 483-487.

(18) Young-Sang, C.; Gi-Ra, Y.; Shin-Hyun, K.; Pine, D. J.; SeungMan, Y. Colloidal Clusters of Microspheres from Water-in-Oil Emulsions. Chem. Mater. 2005, 17, 5006-5013.

(19) Xia, Y.; Yin, Y.; Lu, Y.; McLellan, J. Template-Assisted SelfAssembly of Spherical Colloids into Complex and Controllable Structures. Adv. Funct. Mater. 2003, 13, 907-918.

(20) Zanjani, M. B.; Jenkins, I. C.; Crocker, J. C.; Sinno, T. Colloidal Cluster Assembly into Ordered Superstructures via Engineered Directional Binding. ACS Nano 2016, 10, 11280-11289.

(21) Ngo, T. T.; Liddell, C. M.; Ghebrebrhan, M.; Joannopoulos, J. D. Tetrastack: Colloidal diamond-inspired structure with omnidirectional photonic band gap for low refractive index contrast. Appl. Phys. Lett. 2006, 88, 241920.

(22) Hynninen, A.-P.; Thijssen, J. H.; Vermolen, E. C.; Dijkstra, M.; Van Blaaderen, A. Self-Assembly Route for Photonic Crystals with a Bandgap in the Visible Region. Nat. Mater. 2007, 6, 202-205.

(23) Hynninen, A.-P.; Filion, L.; Dijkstra, M. Stability of LS and $\mathrm{LS}_{2}$ Crystal Structures in Binary Mixtures of Hard and Charged Spheres. J. Chem. Phys. 2009, 131, 064902.

(24) Ducrot, E.; He, M.; Yi, G.-R.; Pine, D. J. Colloidal Alloys with Preassembled Clusters and Spheres. Nat. Mater. 2017, 16, 652-657.

(25) Zhu, J. H.; Liu, C. T.; Pike, L. M.; Liaw, P. K. A Thermodynamic Interpretation of the Size-Ratio Limits for Laves Phase Formation. Metall. Mater. Trans. A 1999, 30, 1449-1452.

(26) Edwards, A. R. The Lattice Dimensions of the $\mathrm{AB}_{2}$ Laves Phases. Metall. Trans. A 1972, 3, 1365-1372.

(27) Kowalik, M.; Tretiakov, K.; Wojciechowski, K. On Relative Stability of Selected Hard Tetramer Solids. Computational Methods in Science and Technology 2010, 16, 141-146.

(28) de las Heras, D.; Schmidt, M. Sedimentation Stacking Diagram of Binary Colloidal Mixtures and Bulk Phases in the Plane of Chemical Potentials. J. Phys.: Condens. Matter 2015, 27, 194115.
(29) de las Heras, D.; Doshi, N.; Cosgrove, T.; Phipps, J.; Gittins, D. I.; van Duijneveldt, J. S.; Schmidt, M. Floating Nematic Phase in Colloidal Platelet-Sphere Mixtures. Sci. Rep. 2012, 2, 789.

(30) Parrinello, M.; Rahman, A. Crystal Structure and Pair Potentials: A Molecular-Dynamics Study. Phys. Rev. Lett. 1980, 45, 1196-1199.

(31) Filion, L.; Marechal, M.; van Oorschot, B.; Pelt, D.; Smallenburg, F.; Dijkstra, M. Efficient Method for Predicting Crystal Structures at Finite Temperature: Variable Box Shape Simulations. Phys. Rev. Lett. 2009, 103, 188302. 